Joël Noret, Pierre Petit, Mort et dynamiques sociales au Katanga (République démocratique du Congo)

Paris, L'Harmattan, Musée royal de l'Afrique centrale, 2011, 159 p.

Maixant Mebiame Zomo

(2) OpenEdition

Journals

Édition électronique

URL : http://journals.openedition.org/assr/24669

DOI : $10.4000 /$ assr.24669

ISSN : $1777-5825$

Éditeur

Éditions de l'EHESS

Édition imprimée

Date de publication : 30 décembre 2012

Pagination : 245

ISSN : 0335-5985

Référence électronique

Maixant Mebiame Zomo, « Joël Noret, Pierre Petit, Mort et dynamiques sociales au Katanga

(République démocratique du Congo) », Archives de sciences sociales des religions [En ligne], 1601

octobre-décembre 2012, mis en ligne le 21 mars 2013, consulté le 21 septembre 2020. URL : http:// journals.openedition.org/assr/24669; DOI : https://doi.org/10.4000/assr.24669

Ce document a été généré automatiquement le 21 septembre 2020.

(c) Archives de sciences sociales des religions 


\section{Joël Noret, Pierre Petit, Mort et dynamiques sociales au Katanga (République démocratique du Congo)}

Paris, L'Harmattan, Musée royal de l'Afrique centrale, 2011, 159 p.

Maixant Mebiame Zomo

\section{RÉFÉRENCE}

Joël Noret, Pierre Petit, Mort et dynamiques sociales au Katanga (République démocratique du Congo), Paris, L'Harmattan, Musée royal de l'Afrique centrale, 2011, $159 \mathrm{p}$. 
1 Cet ouvrage de Joël Noret et Pierre Petit, respectivement professeur assistant à l'Université Libre de Bruxelles et chercheur qualifié au Fond National pour la Recherche Scientifique (FNRS) en Belgique, aborde la question de la mort au Katanga, en République démocratique du Congo (ex-Zaïre). Au regard des nombreux travaux déjà effectués sur le même thème

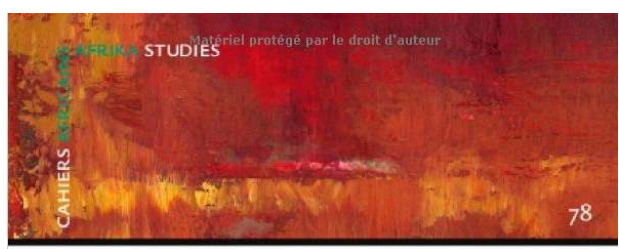

Mort et dynamiques sociales au Katanga (République démocratique du Congo) par les deux auteurs (voir la bibliographie du livre) - Joël Noret vient de publier un ouvrage sur Deuil et funérailles dans le Bénin méridional. Enterrer à tout prix, Édition de l'Université de Bruxelles, 2010. En collaboration avec Pierre Petit et d'autres auteurs, il a également cosigné une petite brochure en 2002 sur Kiriyo. Deuil et prise en charge de la mort à Lubumbashi, fruit d'une enquête réalisée par l'observatoire du changement urbain dirigé à l'époque par Pierre Petit -, cette réflexion se présente donc comme une synthèse de leurs travaux et surtout une comparaison de deux corpus de matériaux ethnographiques de deux sites de recherche différents.

2 Les premières données de terrain qui alimentent la discussion dans cet ouvrage ont été collectées entre 1988 et 1996 par Pierre Petit sur les rites funéraires "traditionnels » des Luba du Katanga, dans le cadre de la rédaction de sa thèse de doctorat. La deuxième série de données ethnographiques a été collectée dans la ville de Lubumbashi dont une partie de la population est composée des Luba du Katanga. Aussi, le choix d'une étude en milieu rural (Katanga), puis en milieu urbain (Lubumbashi) peut s'expliquer ici par le fait que les auteurs appréhendent le monde rural comme celui du «traditionnel » et où l'on peut facilement saisir les funérailles, le deuil et les représentations sociales de la mort avant l'influence du christianisme et de la colonisation. Selon une certaine épistémologie, les auteurs ont mis entre parenthèses les changements internes et externes induits par l'ordre social de la colonie et de la postcolonie. Comment donc faire pour remonter le temps et recueillir des données ethnographiques de cette société Luba d'avant sa mutation? Pour cela, les auteurs ont pratiqué une anthropologie rétrospective en adoptant une "ethnographie des "vieux hommes" ou, alternativement, des "dames", ciblées explicitement sur l'époque où le christianisme n'avait pas le rôle hégémonique qu'il possède maintenant » (p.14). Toute la difficulté dans ce genre d'exercice généralement admise dans les sciences sociales réside dans le contournement des problèmes de mémoires des interlocuteurs. En effet, inévitablement les témoignages des personnes âgées sont souvent incomplets et contiennent des erreurs. Les souvenirs n'ont pas toujours des contours nettement dessinés.

3 À l'inverse, les enquêtes en ville (Lubumbashi), synonyme de modernité, insistent davantage sur les tensions, les évolutions récentes et la pluralité des structures en 
concurrence et qui gèrent la mort. Autrement dit, le problème posé dans cet ouvrage est de comprendre, puis d'analyser le deuil, les funérailles et les représentations sociales de la mort dans la société luba traditionnelle et de repérer les changements et les évolutions de ces pratiques et ses représentations. Le meilleur moyen de saisir ses évolutions est de s'intéresser à la ville de Lubumbashi. Pour soutenir cette démonstration, l'argumentaire repose sur une structuration du livre en cinq chapitres très inégalement structurés et précédés d'une introduction qui précise clairement les enjeux théoriques de la recherche et surtout l'orientation comparative de la méthodologie.

4 Le premier chapitre nous introduit d'emblée dans la société luba de près d'un million et demi à deux millions d'individus qui habitent un territoire vaste d'une superficie de cent mille $\mathrm{km}^{2}$. Le point central de ce chapitre est de savoir comment s'organise cette société lorsqu'un de ses membres trouve la mort. La réponse est simple : la société luba prend la mort en charge. De l'annonce du décès de la personne aux différents rites de veuvage via l'enterrement, les auteurs de ce livre nous décrivent fort justement l'organisation ou la réaction de la société luba dont l'ordre se trouve déstabilisé par la mort d'un de ses membres. En effet, prendre en charge la mort revient tout d'abord à la société à gérer les modalités de l'annonce du décès, moment de tensions et de conflits entre la famille endeuillée et celle de l'époux, accusé d'être le responsable quelle que soit la cause réelle du décès puisque, selon la tradition luba, par le mariage, l'époux devient automatiquement responsable de tout ce qui pourrait arriver à sa femme, $\mathrm{y}$ compris de sa mort. Très souvent, c'est l'un de ses frères ou un autre parent qui se charge d'annoncer le décès. De préférence, on désigne un bon coureur qui peut échapper aux sévices et châtiments qu'il peut subir en allant annoncer le décès ( $k w e l a$ umpanzi) à la belle-famille. L'expression kwela umpanzi consacrée pour référer à l'annonce du décès d'une femme désigne aussi des ustensiles emblématiques de la cuisine utilisés par les femmes. Le corps de la femme est ensuite accompagné à son village natal où le veuf est tenu d'expliquer les circonstances du décès. La famille de la défunte s'occupe du corps de leur fille (traitement, maquillage, etc.), fixe le montant de la compensation funéraire en accord avec la famille du gendre et détermine le jour de l'enterrement «qui avait lieu en général le lendemain du décès après une veillée funèbre. On enterrait de préférence le matin, quand il n'y avait pas de vent, de peur que la brise ne sépare le corps et l'esprit de la morte [...] Les chefs et les dignitaires étaient enterrés nuitamment » (p. 26).

5 À l'inverse, une épouse n'est pas tenue responsable du décès de son époux. La mort de ce dernier ne déclenche pas de frictions entre les familles alliées. Un messager se charge simplement de prévenir la belle-famille et ils se retrouvent ensemble un peu plus tard pour pleurer le gendre. La famille du défunt se charge de gérer la mort de leur enfant sans tension ou conflit avec la belle-famille comme c'est le cas lorsqu'il s'agit de la mort d'une femme. On peut souligner et regretter que la mort dont il est surtout question dans cet ouvrage est celle qui concerne deux cas de figure : d'une part le décès d'un homme marié et d'autre part celui d'une femme également mariée; et, dans les deux cas, tous vivant généralement ensemble sous le même toit. Les auteurs décrivent et analysent la réaction de la société luba par rapport à ces deux formes de décès. Cependant, comment cette même société s'organise-t-elle lorsqu'il s'agit de la mort d'un enfant, d'un célibataire, d'un dignitaire ou d'un chef, etc.? Il est clair que tout décès d'un individu, quels que soient son rang et son statut dans la société appelle à une organisation de cette dernière. Ce chapitre riche en description des différentes 
traditions rituelles de la mort présente aussi la période de l'observation du deuil qui précède l'enterrement. Deux moments caractérisent cette phase. Le premier deuil (ntanda) se caractérise par les lamentations, le rite du veuvage très sévère observé par l'époux et la seconde phase dite Kusuma est une sorte d'amende (mishinga) payée par le veuf dès son retour du cimetière. Cette phase du deuil correspond aussi au retour du veuf à son domicile en compagnie des membres de sa belle-famille. Ici, le veuf peut jouir de certains droits élémentaires dont le décès de son épouse l'avait privé (manger, fumer, etc.).

6 La prise en charge des morts à Lubumbashi (chap. II) fait intervenir de nouveaux acteurs, lieux et surtout une déritualisation du deuil quand on compare ses modalités au pays luba rural. En effet, les pratiques du deuil en ville sont largement définies selon un cadre symbolique chrétien (chap. V) qui estompe le pouvoir des morts; un espace urbain où se dessine une nouvelle géographie de la mort impliquant la morgue, l'église et le cimetière, et enfin, une temporalité citadine qui limite la disponibilité de chacun pour le temps du deuil. Les auteurs décrivent certaines évolutions observées en ville par rapport à l'ordre ancien luba. L'annonce du décès par exemple ne se fait plus selon la démarche ancienne. Les communiqués radio et presse ou encore des courriers ont remplacé le messager qui allait annoncer le décès. De même, l'enterrement qui se faisait systématiquement le lendemain du décès et très tôt le matin se déroule plusieurs jours après. Ce qui permet de réunir suffisamment d'argent et surtout d'attendre la présence de certains membres de la famille partis en voyage ou vivant dans d'autres villes ou pays. Les morgues et leurs professionnels ont pris le relais dans le traitement du corps en lieu et place de la famille. Autrement dit, entre le monde rural luba et celui de Lubumbashi, on observe que la famille ou la parenté en général perd le monopole sur l'organisation des obsèques et du deuil. Si dans la société traditionnelle luba la parenté prenait une place très importante et chacun par sa position au sein de la famille (oncle, neveu, fils, etc.) jouait un rôle précis, en ville, il y a un changement majeur avec l'éclosion d'un marché professionnel de la mort (commerçants spécialisés en matériels ou en services funéraires: fleuristes, fabricants de cercueils, entrepreneurs de pompes funèbres, morguiers, chorales avec orchestres, etc.), les amis et connaissances qui viennent en aide et également l'employeur si le défunt travaillait dans une entreprise ou l'administration publique.

7 Le religieux versus christianisme pentecôtiste occupe également une place très importante dans cette étude sur la mort. L'auteur consacre le dernier chapitre - le chapitre $\mathrm{V}$ - à cet idiome religieux qui a largement contribué à façonner ou remodeler la culture funéraire urbaine et les modes de présence des défunts. Le pentecôtisme diabolise les rites traditionnels liés aux morts et réfute l'idée d'une communication entre les vivants et les morts. Cependant, comme de nombreux travaux avant celui-ci, les auteurs soulignent le paradoxe et les ambiguïtés du pentecôtisme qui diabolise les rites traditionnels liés aux morts, mais s'approprie cet imaginaire traditionnel et les retraduit à sa façon. Aussi, chez les fidèles, même si on se considère comme chrétien, on pense que les rites traditionnels sont plus efficaces et on y a toujours recours. 